\title{
Horizontal dispersion of near-inertial oscillations in a turbulent mesoscale eddy field
}

\author{
by Patrice Klein ${ }^{1}$ and Stefan Llewellyn Smith ${ }^{2}$
}

\begin{abstract}
We study the dispersion of wind-induced near-inertial oscillations (NIOs) in a fully turbulent baroclinic mesoscale eddy field characterized by a continuous wavenumber spectrum. The influence of the eddy field on the horizontal dispersion of the different NIO modes is analyzed using a vertical normal mode expansion. Previous studies have identified two dispersion regimes: trapping and strong dispersion. We examine the modes in physical and spectral space to assess which regime prevails.

Numerical and analytical results show the prevalence of a trapping regime. For each NIO mode, there exists a critical horizontal wavenumber, $k_{c}$, that separates large-scale NIO structures, where trapping dominates, from the much less energetic small-scale NIO structures, where strong dispersion dominates. The maximum efficiency of dispersion for scales close to $k_{c}$ concentrates NIO kinetic energy at these scales.

The wavenumber $k_{c}$ results from a balance between refraction and dispersion. This balance first occurs at the highest wavenumber. Thereafter, $k_{c}$, which has dimensional expression $k_{c}^{2}=$ $\pi /\left(f t R_{m}^{2}\right)$, decreases with time at a rate inversely proportional to the radius of deformation, $R_{m}$, of the baroclinic NIO mode considered. As a consequence, at any given time, higher NIO baroclinic mode energy can mostly be found in small-scale negative vorticity structures, such as filaments near sharp vorticity fronts, whereas lower NIO mode energy is concentrated within the core of mesoscale anticyclonic vortices. For large times, a saturation mechanism stops the time-evolution of $k_{c}$ at a value close to the peak of the kinetic energy spectrum of the QG flow field.
\end{abstract}

\section{Introduction}

Near-inertial oscillations (NIOs), that is internal waves with frequencies close to the Coriolis frequency, are prominent features of the upper ocean (Webster, 1968). They can transport the energy input by storms from the surface mixed layer into the water column (D'Asaro et al., 1995) and may represent an important contributing factor for mixing in the ocean, since the vertical shear they induce can reduce the Richardson number below 0.25 and trigger mixing events. Considerable observational evidence (see for example Weller, 1982; Kunze and Sanford, 1984; D'Asaro et al., 1995) has emphasized the spatial

1. Laboratoire de Physique des Océans, IFREMER/Centre de Brest, B.P. 70, 29280 Plouzane, France. email: pklein@ifremer.fr

2. Department of Mechanical and Aerospace Engineering, University of California, San Diego, California, 92093, U.S.A. 
modulation of their horizontal and vertical propagation by mesoscale structures, and this has led to much theoretical work in the last 15 years (Kunze, 1985; Wang, 1991; Klein and Treguier, 1995a,b; D'Asaro, 1995a,b; Young and Ben Jelloul, 1997; hereafter YBJ). The primary mechanism responsible for spatial modulation is the refraction of NIOs by the vorticity of the mesoscale eddies (Kunze, 1985) or the planetary vorticity gradient (D'Asaro, 1995a). This refraction can be interpreted as a spatial modulation of the frequency of these waves by the vorticity field, or to be more precise, as a spatial modulation of the intrinsic frequency of the waves with respect to the lower bound of the internal wave band.

Some of the studies devoted to the spatial modulation of NIOs by mesoscale eddies and their subsequent dispersion have investigated their propagation behavior using a raytracing approach (Kunze, 1985). Others have used a vertical normal-mode expansion (Klein and Treguier, 1995a; Balmforth et al., 1998). These studies have considered mostly idealized and isolated barotropic mesoscale structures (characterized by a given length scale) such as a geostrophic jet or an anticyclonic eddy. One important outcome has been the identification of two horizontal dispersion regimes of the NIOs: the 'trapping' regime and the 'strong dispersion' regime. Which regime appears depends on the relative order of magnitude of the refractive and dispersive effects that is given by the ratio of the length scale of the mesoscale structure to the Rossby radius of deformation of the NIO baroclinic mode considered. A large ratio leads to the dominance of the 'trapping' regime identified by Kunze (1985), where NIOs are affected by the local vorticity and disperse slowly. According to WKB theory, the horizontal wavenumber $(k)$ of the NIOs evolves with time as $D k / D t=-\nabla Z$, with $\nabla Z$ the horizontal vorticity gradient. Inertial waves tend to be deflected away from regions of positive vorticity and steered toward regions of negative vorticity. The spatial structure of the NIO field then resembles the vorticity field. On the other hand, a small ratio leads to the dominance of the 'strong dispersion' regime where NIOs disperse quickly and are weakly affected by vorticity. Analytical investigations and numerical simulations have shown that the spatial structure of the NIO field in this regime resembles not the vorticity but the streamfunction (see Klein and Treguier, 1995a; YBJ).

The goal of our study is to extend the results obtained so far for idealized and isolated geostrophic structures to a fully turbulent mesoscale eddy field characterized by a continuous wavenumber spectrum over a large range of horizontal scales. This should allow us to understand the dispersion of NIOs in more realistic situations where mesoscale structures interact strongly; i.e., where the inverse energy cascade and the direct tracer cascade are both significant. Such structures have been well documented in regions such as the Gulf Stream, the Azores Front, and the Antarctic Circumpolar Current, and recent satellite data have revealed their existence in other regions (Stammer, 1997; Wunsch, 1997). We address several specific questions. For a turbulent eddy field, does the 'trapping' regime or the 'strong dispersion' regime dominate? What can be deduced about the spatial structure of different NIO baroclinic modes? In which mesoscale structures is NIO dispersion most efficient? What are the effects of advection relative to refraction and 
dispersion effects? The present study focuses mostly on the horizontal dispersion of different NIO baroclinic modes. Implications for the vertical propagation and on the resulting 3-D distribution of the near-inertial waves will be analyzed in a future study. Hence, the present work should be considered as a first step in understanding 3-D NIO dispersion in a fully turbulent mesoscale eddy field.

In this study, we take advantage of the YBJ framework to describe propagation of NIOs in a turbulent mesoscale eddy field. This approach, unlike WKB approaches, does not assume an a priori horizontal scale separation between the NIOs and the background flow. Therefore, it is well suited to exploring the dispersion of NIOs embedded in a background flow characterized by a continuous horizontal wavenumber spectrum, which is our main purpose. In the present study the background flow is assumed to be quasi-geostrophic: its Rossby number is small and its Burger number is $O(1)$. The characteristic horizontal scale of the NIOs is the same as that of the background flow but the NIO vertical scale is assumed to be much smaller than that of the mesoscale eddy field (i.e., the NIO Burger number is much smaller than one). With these scalings, YBJ show that inertial waves are recovered to leading order, and that departures from perfect inertial oscillations become appreciable on the slow time scale $t_{s} \equiv\left(\epsilon^{2} f\right)^{-1}$, with $\epsilon^{2}$ the Rossby number and $f$ the inertial frequency. The choice of these scalings allows us to use the simplest formulation of the NIO equations proposed by YBJ. An additional advantage of using the YBJ approach is that the derivation of their equations involves an asymptotic reduction of the problem that filters out the fast inertial period and isolates the slower subinertial evolution of the amplitude. Then their equations can easily be incorporated into a model used to simulate quasi-geostrophic turbulence.

The simplest equation based on the YBJ theory has been incorporated into the quasi-geostrophic spectral model of Hua and Haidvogel (1986). The NIO equation and the quasi-geostrophic model are described in Section 2. Numerical results for the horizontal dispersion of the different NIO modes in both physical and spectral space are described in Section 3. A simple analytical theory to explain these results is proposed in Section 4. Finally, the results of this study are discussed in Section 5, and conclusions and prospects for future work are outlined in Section 6.

\section{Formulation of the NIO dispersion problem}

\section{a. NIO equations and scalings}

Young and Ben Jelloul (1997) consider Boussinesq, inviscid, incompressible and hydrostatic flow and linearize about a background geostrophic flow. The subsequent derivation involves an asymptotic reduction of the linearized primitive equations using a multiple scale expansion in $\epsilon^{2}$ that allows one to recover the slower subinertial evolution of the amplitude of the near-inertial oscillations on the time scale $t_{s} \equiv\left(\epsilon^{2} f\right)^{-1}$ introduced above. To leading order, the NIO velocity field, $(u, v, w)$, buoyancy, $b$, and pressure, $p$, are expressed in terms of a complex field, $A(x, y, z, t)$, via 


$$
\begin{gathered}
u+i v=e^{-i f_{0} t \mathscr{L} A}, \\
w=-1 / 2 f_{0}^{2} N^{-2}\left(A_{z x}-i A_{y z}\right) e^{-i f_{0} t}+c . c . \\
b=\frac{i}{2} f_{0}\left(A_{z x}-i A_{y z}\right) e^{-i f_{0} t}+c . c ., \\
p=\frac{i}{2} f_{0}\left(A_{x}-i A_{y}\right) e^{-i f_{0} t}+c . c .
\end{gathered}
$$

with $i$ complex unity and $f=f_{0}+\beta y$ the inertial frequency. $N(z)$ is the buoyancy frequency and $\mathscr{L}$ a differential operator defined by

$$
\mathscr{L A}=\left(f_{0}^{2} N^{-2} A_{z}\right)_{z} .
$$

Following Flierl (1978) and Gill (1982), if boundary conditions are rigid lids at top $(z=0)$ and bottom $(z=-H)$, normal vertical modes can be defined as solutions of the Sturm-Liouville problem:

$$
\mathscr{L} g_{m}(z)=-\frac{1}{R_{m}^{2}} g_{m}(z)
$$

with $g_{m}$ the eigenfunctions and $1 / R_{m}^{2}$ the eigenvalues. $R_{m}$ is the internal Rossby radius of deformation associated with the $m$ th baroclinic mode. The NIO kinetic energy $\left(u^{2}+v^{2}\right)$ can be retrieved directly from the slower subinertial amplitude $\mathscr{L} A$ by

$$
u^{2}+v^{2}=\mathscr{L} A \mathscr{L} A^{*}=|\mathscr{L} A|^{2} .
$$

with $*$ denoting the complex conjugate.

Using this approximation, the NIO equation derived by YBJ in the presence of a background QG flow takes the dimensional form

$$
\frac{\partial}{\partial t} \mathscr{L} A+J(\psi, \mathscr{L} A)+\frac{i f_{0}}{2} \nabla^{2} A+i\left(\beta y+\frac{Z}{2}\right) \mathscr{L A}=0,
$$

where $\psi$ and $Z \equiv \nabla^{2} \psi$ are respectively the streamfunction and relative vorticity of the QG flow, and $J$ the Jacobian. The boundary conditions in the vertical are that $A_{z}$ vanish at the top and bottom of the domain. One important aspect of (8) is that the integral of $|\mathscr{L A}|^{2}$ over the spatial domain is conserved (see Metzger, 1999).

The second term in (8) corresponds to advection by the geostrophic flow, the third to dispersion and the fourth to refraction. The terms 'advection,' 'dispersion' and 'refraction' will be frequently used in what follows, and generally refer to the effect of the different terms in the governing NIO equation.

The $\beta$-effect is expected to play a role in the propagation of NIO energy. However, the mesoscale eddy field considered in this study is such that the RMS amplitude of $Z$ is large compared to the $\beta$-effect. Simulations performed with nonzero $\beta$ in (8) have confirmed its 
weak influence on NIO dispersion compared to that of $Z$. Since these are small effects, the $\beta$ term has been set to zero in this study.

The derivation of (8) by YBJ makes use of a scaling analysis where the horizontal length scale $L$ and time scale $T \equiv L / U$ of the NIOs are assumed to be the same as for the background QG flow ( $U$ is the velocity scale of the QG flow). On the other hand, the vertical scale of the NIOs, $\lambda_{V}$, is assumed to be much smaller than that of the QG flow. More precisely $\lambda_{V} \approx \epsilon H$ with $\epsilon^{2}=U /\left(f_{0} L\right)$. Since the QG approximation means that stretching and relative vorticity terms have the same order of magnitude (i.e., $N_{0}^{2} H^{2}$ I $\left.f_{0}^{2} L^{2}=O(1)\right)$ the terms in (8), whose amplitudes are in the ratio $1: 1: N_{0}^{2} \lambda_{V}^{2} / f_{0} L U: 1$, scale identically in nondimensional units. YBJ show that, with this choice of the NIO vertical scaling (which corresponds to $q=2$ in their terminology), the vertical variation of the QG flow is incorporated into (8).

Using the preceding scalings, with tildes denoting nondimensional quantities, the NIO equations become:

$$
\frac{\partial}{\partial t} \tilde{\mathscr{L}} A+\tilde{J}(\tilde{\Psi}, \tilde{\mathscr{L}} A)+\frac{i}{2} \tilde{\nabla}^{2} A+\frac{i}{2} \tilde{Z} \tilde{\mathscr{L}} A=-\nu \tilde{\nabla}^{4} \tilde{\mathscr{L}} A .
$$

with

$$
\mathscr{L}=\tilde{\mathscr{L}} /\left(\epsilon^{2} L^{2}\right)
$$

To ensure consistency with the equations of motion which contain dissipative terms, appropriate dissipative terms have been added in (9). These can be deduced using the procedure of YBJ. Their effect is small, but they are necessary both for consistency with the flow equations and for numerical stability for large-duration integrations. We use a hyperviscosity operator to be consistent with the parameterization of dissipative terms in the QG flow equations.

\section{b. Vertical normal mode analysis}

From now on, tildes are dropped. The equation for $\mathscr{L} A$ is solved after expanding in vertical normal modes, using

$$
\mathscr{L} A(x, y, z, t)=\sum_{0}^{\infty} \mathscr{L} A_{m}(x, y, t) g_{m}(z)=-\sum_{1}^{\infty} \lambda_{m}^{2} A_{m}(x, y, t) g_{m}(z),
$$

with $g_{m}$ and $\lambda_{m}^{2}$, respectively, the eigenfunctions and eigenvalues of the operator $\mathscr{L}$. The eigenvalue $\lambda_{m}^{2}$ is related to the internal Rossby radius of deformation $\left(R_{m}\right)$ of the $m$ th vertical mode through: $\lambda_{m}^{2}=\epsilon^{2} L^{2} / R_{m}^{2}$. The barotropic component is zero (i.e., $\mathscr{L} A_{\mathrm{O}}=0$ ), so all NIO modes are baroclinic. Then the $\mathscr{L} A$ equation in vertical modes becomes:

$$
\frac{\partial \mathscr{L} A_{m}}{\partial t}+\sum_{j, k} e_{m, j, k} J\left(\psi_{j}, \mathscr{L} A_{k}\right)-\frac{i}{2} \frac{\nabla^{2} \mathscr{L} A_{m}}{\lambda_{m}^{2}}+\frac{i}{2} \sum_{j, k} e_{m, j, k} Z \mathscr{L} A_{k}=-\nu \nabla^{4} \mathscr{L} A_{m},
$$


where

$$
e_{m, j, k}=\frac{1}{H} \int_{-H}^{0} g_{m}(z) g_{j}(z) g_{k}(z) d z
$$

is the triple-interaction coefficient between modes $m, j$ and $k$ (defined in Hua and Haidvogel, 1986). These triple-interaction coefficients express the nonlinear energy transfer between the NIO baroclinic modes. Their values depend strongly on $N^{2}(z)$.

It should be noted that vertical critical layers for NIOs may arise when the change with depth of the QG vorticity or velocity is comparable or greater than the deviation of the wave's intrinsic frequency from the Coriolis frequency (Kunze, 1985). In that case the expansion (11) is not convergent and the use of a finite normal mode expansion is not valid. From (12), such critical layers may arise through the nonlinear energy transfer between baroclinic modes in the second term (involving the QG velocity) and in the fourth term (involving the QG vorticity). However, we assume in this study that these nonlinear transfers are negligible which prevents the appearance of vertical critical layers, at least on the time scales considered here, and allows the use of a finite normal mode expansion.

Indeed the chosen $N^{2}(z)$ profile corresponds to a summertime stratification with the initial NIO distribution trapped within a shallow surface mixed layer $(\approx 30 \mathrm{~m}$ thick $)$ whereas the QG flow is forced by the instability of a large-scale mean current shear whose zero crossing is at $1500 \mathrm{~m}$. As a result, the QG flow is principally captured by the barotropic and the first baroclinic modes while the NIO modes concern much higher baroclinic modes; i.e., the indices $m$ and $k$ in (12) are much larger than $j$. We have checked that in this situation the corresponding triple-interaction coefficients (defined by (13)) are negligible when $m \neq k$. Thus, when $j=1$ and $k=6, e_{m, j, k} \approx 1$ when $m=k$ but $e_{m, j, k} \approx$ 0 when $m \neq k$. This ensures that the corresponding nonlinear energy transfers between different NIO baroclinic modes are negligible, which prevents the appearance of critical layers, at least on short time scales. This checking has also been done by comparing results from simulations using both (12) and the following equation

$$
\frac{\partial \mathscr{L} A_{m}}{\partial t}+J\left(\psi, \mathscr{L} A_{m}\right)-\frac{i}{2} \frac{\nabla^{2} \mathscr{L} A_{m}}{\lambda_{m}^{2}}+\frac{i}{2} Z \mathscr{L} A_{m}=-v \nabla^{4} \mathscr{L} A_{m},
$$

where the values of the QG velocity and vorticity fields (assumed to be vertically homogeneous) are those at $40 \mathrm{~m}$. The results obtained from both sets of simulations are almost identical for a simulation duration of 15 days. Thus, Eq. (14) can be considered a valid first approximation for the study of the behavior of the different NIO modes. This equation is equivalent to assuming that the QG flow felt by the NIOs is barotropic. Then the only difference in the evolution of the NIO modes comes from their Rossby radius of deformation $R_{m}$ (i.e., $\lambda_{m}$ in (14)).

At last, the fact that the NIO baroclinic modes are much higher than the QG modes is consistent with the assumption considered before about the NIO vertical scales. Indeed if 
we consider the relation $R_{m}^{2} / R_{1}^{2}=O\left(\epsilon^{2}\right)$, this implies that the NIO vertical scales are much smaller than that of the QG flow (i.e., $\lambda_{v} \approx \epsilon H$ from the preceding analysis) since these vertical scales are linked to the internal Rossby radii through $f / N$ (Gill, 1982).

\section{Numerical simulations}

\section{a. The $Q G$ flow and the initial NIO field}

The buoyancy profile adopted is that of Klein et al. (1998), with the addition of a $30 \mathrm{~m}$ thick surface mixed layer above a $15 \mathrm{~m}$ thick seasonal thermocline. This matches conditions encountered during summer (see also Klein and Treguier, 1995a). As in Klein et al. (1998), the QG flow is forced by the baroclinic instability of a vertically sheared zonal flow $\bar{U}(z)$ (captured by the first baroclinic mode whose zero crossing is at $1500 \mathrm{~m}$ ) and damped by a bottom Ekman layer (at $z=-H$ ) parameterized with a friction coefficient. The governing equation for the background flow is

$$
\frac{\partial q}{\partial t}+\bar{U} \frac{\partial q}{\partial x}+J(\phi, q)+\beta \frac{\partial \phi}{\partial x}=M+D,
$$

where $\phi$ is the perturbed QG streamfunction related to $\psi$ by $\psi=-\bar{U} y+\phi . q \equiv \nabla^{2} \phi+$ $\left(f_{0}^{2} N^{-2} \phi_{z}\right)_{z}$ is the perturbed potential vorticity, $M$ the forcing term and $D$ the dissipation term. The nondimensionalization of the potential vorticity equation uses the length-scale $L=350 \mathrm{~km}$ associated with the domain size. This length-scale is chosen to correspond to 7 Rossby radii in the nondimensional domain $(0,2 \pi)$. The velocity scale is $U=$ $0.12 \mathrm{~m} \mathrm{~s}^{-1}$ and the time scale is the advective time $T=L / U=33.8$ days. The Coriolis frequency $f_{0}$ enters the problem only through the first internal Rossby radius $R_{1}=50 \mathrm{~km}$. The vertical scale is $H=5400 \mathrm{~m}$. A basic run at $256 \times 256$ resolution with 16 vertical modes was carried out to obtain a turbulent mesoscale eddy field in statistical equilibrium. Figure 1 shows the streamfunction and vorticity fields of the QG flow at a depth of $50 \mathrm{~m}$ at the end of this run after 3.3 years. The QG flow is mostly captured by the barotropic and the first baroclinic mode (whose zero crossing is at $1500 \mathrm{~m}$ ), and hence this flow is essentially homogeneous in the vertical from the surface down to $400 \mathrm{~m}$. This simulated turbulent quasi-geostrophic flow is similar to that described in Klein et al. (1998), which indicates that the additional mixed layer has no effect on this flow.

The vorticity field (Fig. 1b) displays the usual emergence of small-scale coherent vortices and strong vorticity fronts as well as a large number of filaments. Figure 1c shows the vorticity spectrum at $100 \mathrm{~m}$. This spectrum is characterized by a spectral peak at $k=5$ and a $k^{-1.5}$ spectral slope. This means that the most energetic vorticity gradients are captured by the smallest scales. Since the relative vorticity is the Laplacian of the streamfunction, the streamfunction spectrum has a $k^{-5.5}$ slope, which explains the dominance of large scales in this field (Fig. 1a). This QG flow field is used as the initial QG flow for the integration of the NIO equation.

The initial NIO distribution is given by the large-scale disturbance: 
(a) PHI at $z=100 \mathrm{~m}$ and $t=0$. days

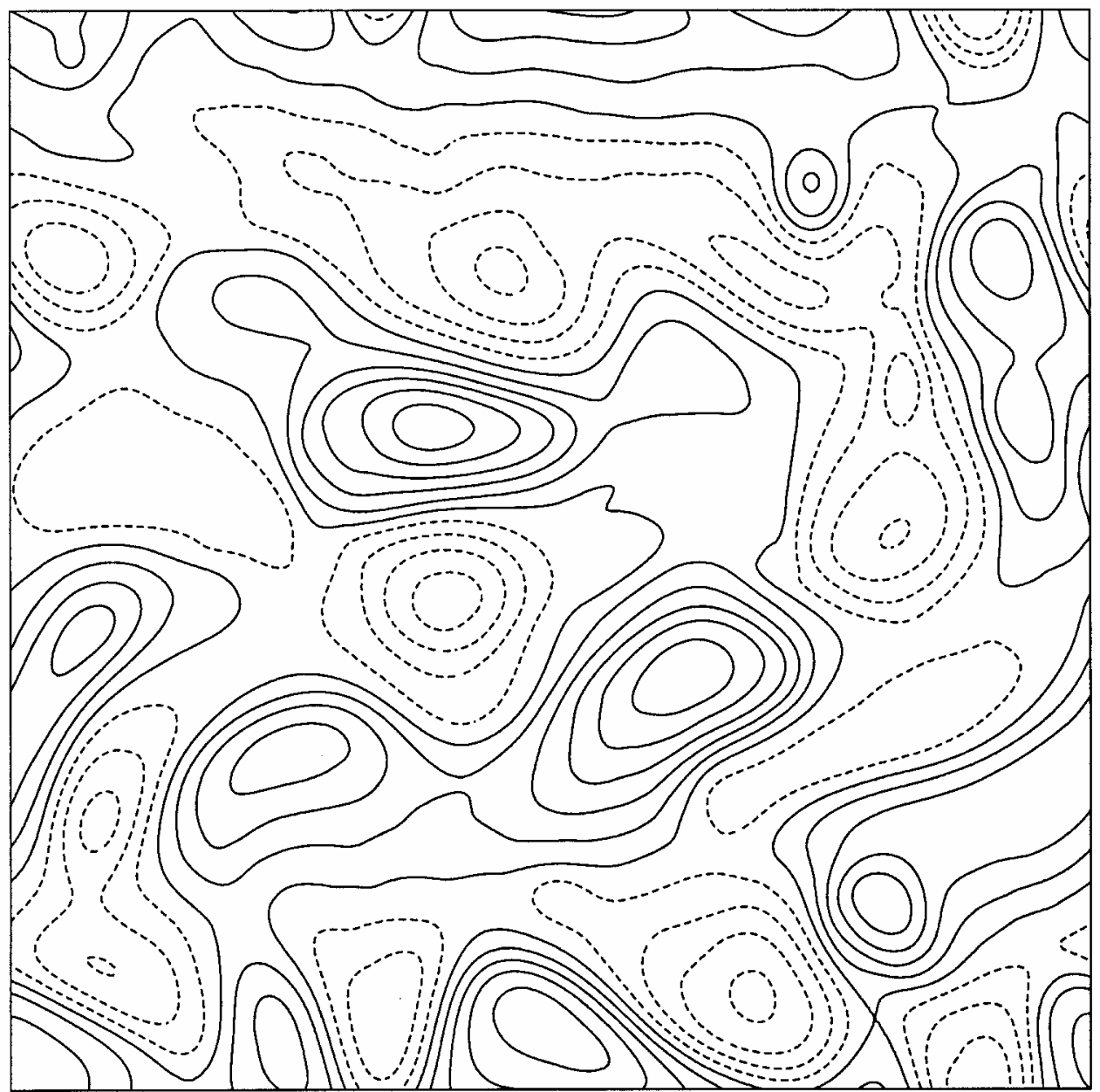

\section{CONTOUR FROM -2 TO 2.5 BY .5}

Figure 1. Contour maps (a, b) and spectrum (c) of the initial streamfunction (a) and vorticity (b, c) fields at a depth of $100 \mathrm{~m}$. In $(\mathrm{a}, \mathrm{b})$ dashed and continuous contours correspond, respectively, to negative and positive values relative to the mean.

$$
\mathscr{L} A_{m}(x, y, 0)=u_{m}(\sin x+i \cos x),
$$

which corresponds to a horizontally homogeneous NIO kinetic energy field. To verify that the chosen summertime stratification leads to a decoupling between the NIO and QG vertical modes, we consider an initial NIO disturbance trapped within a $30 \mathrm{~m}$ thick surface mixed layer, a situation close to that used by Wang (1991), Klein and Treguier (1995a) and Balmforth et al. (1998). The resulting normal-mode analysis displays that the NIO field is 
(b) $\mathrm{Z}$ at $\mathrm{z}=100 \mathrm{~m}$ and $\mathrm{t}=0$. days

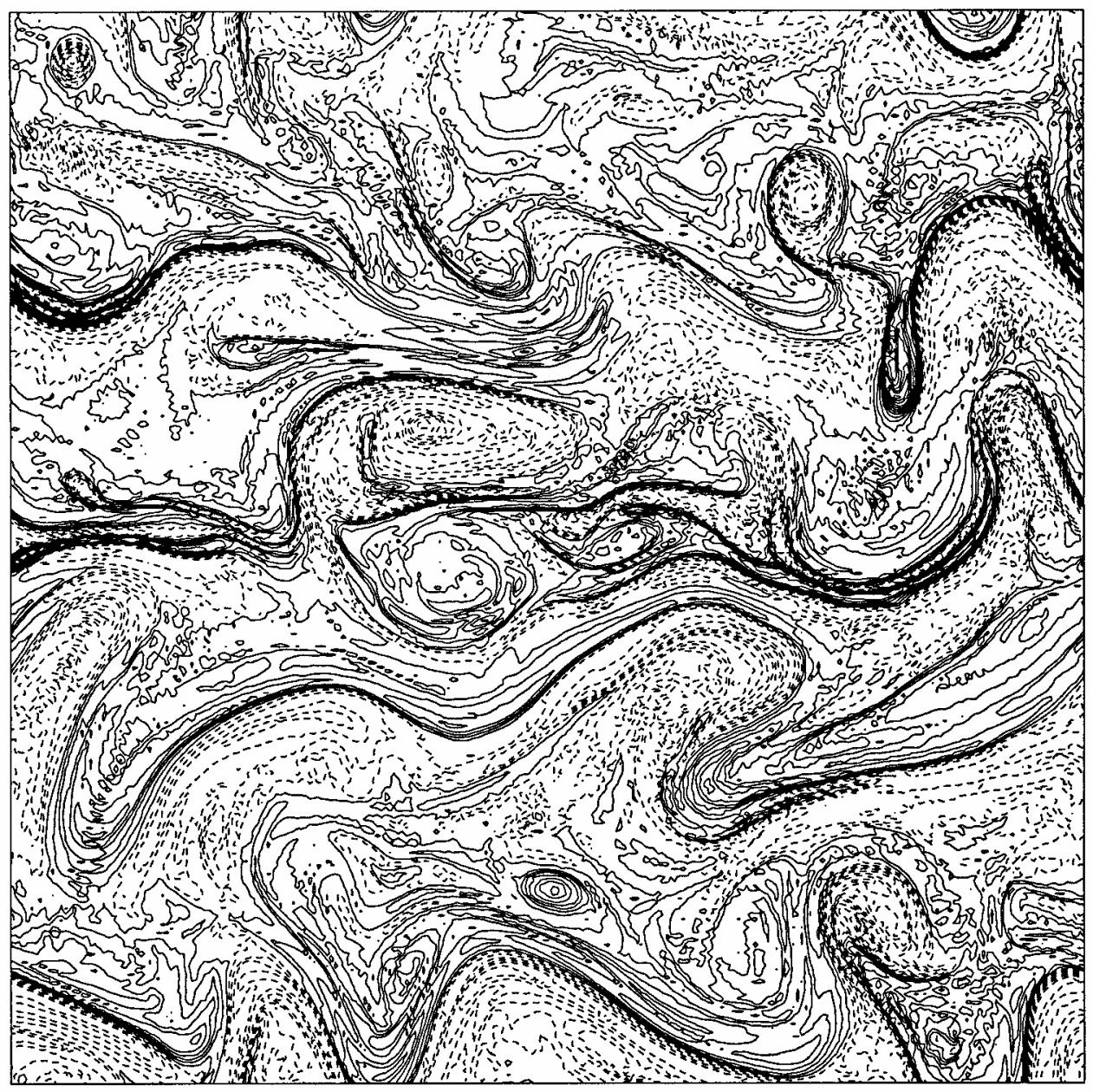

CONTOUR FROM -100 TO 120 BY 10

Figure 1. (Continued)

mostly captured by the 6th baroclinic mode. This indicates that the vertical scale of the NIOs is much smaller than that of the QG flow.

Since this is a process study, the NIO modes have been nondimensionalized so that the modes initially have not only the same spatial distribution but also the same amplitude; i.e., $u_{m}=1$ for all $m$ in (16). This choice allows us to compare directly the behavior of different NIO modes and examine the mechanisms involved. From these initial conditions, since (14) is linear in terms of NIO modes, the behavior of the modes now only differs because of the value of $\lambda_{m}$. Subsequent runs involve NIO Eq. (14) integrated in time along with the evolving background QG flow. 


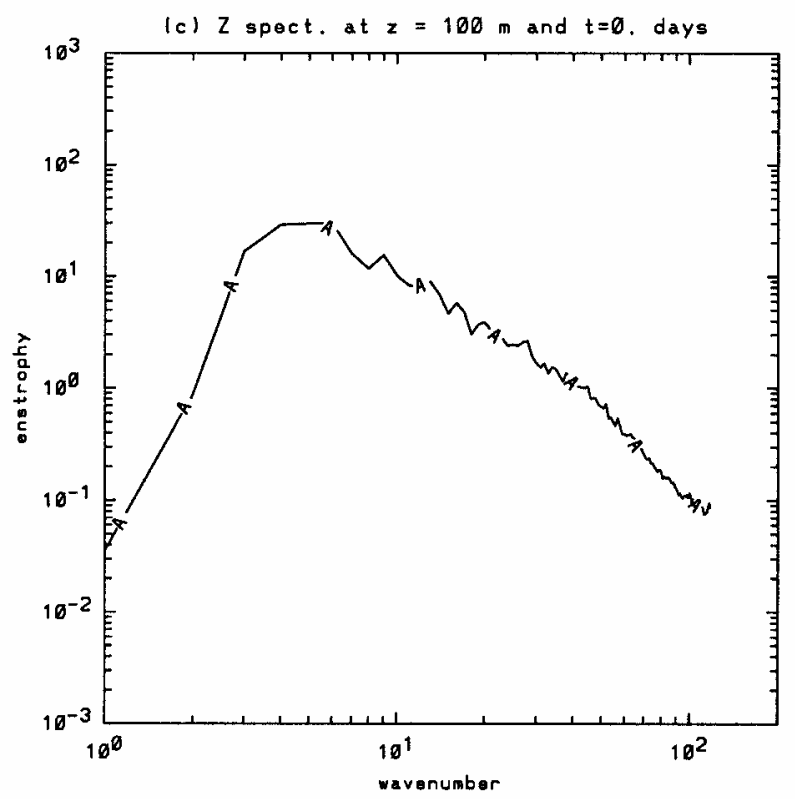

Figure 1. (Continued)

\section{b. Horizontal dispersion of the NIO baroclinic modes}

In order to characterize the evolution of the modes, we examine the spatial distribution and spectra of both the NIO subinertial amplitude, $\mathscr{L} A_{m}$ and the NIO speed, $\sqrt{u_{m}^{2}+v_{m}^{2}}=$ $\left|\mathscr{L} A_{m}\right|$, of the different modes. The NIO amplitude is the most important quantity to investigate since the total kinetic energy, which involves all the modes, requires the use of first (11) and then (7). However, the NIO speed, $\left|\mathscr{L} A_{m}\right|$, leads to a better characterization of horizontal dispersion of the different modes, in particular of the spatial distribution of the kinetic energy associated with these modes.

Comparing Figure $1 \mathrm{~b}$ and Figure 2 reveals a very weak evolution of the vorticity field over 7 days. On the other hand, the different NIO modes exhibit significant changes, which differ between modes, despite identical initial distributions. To compare the evolution of the lower and higher modes, we examine the spatial distribution of modes $m=3$ and 7 (whose Rossby radius are, respectively, 16 and $5 \mathrm{~km}$ ) after 7 days of simulation. Figure 3a shows the imaginary part of the amplitude of mode 3 . Its spatial distribution is similar to that of its real part (not shown). It is characterized by patchy mesoscale structures which are almost isotropic and whose extrema are principally located within mesoscale eddies. The spatial variability of these structures matches the vorticity field more closely than the streamfunction field. The NIO speed distribution of this mode (Fig. 3b) reveals highly energetic structures, whose shape is slightly more stretched, with maxima mostly located within anticyclonic eddies.

The NIO amplitude distribution of the higher modes (mode 7 on Fig. 4a) is characterized 
$\mathrm{Z}$ at $\mathrm{z}=100 \mathrm{~m}$ and $\mathrm{t}=7$. days

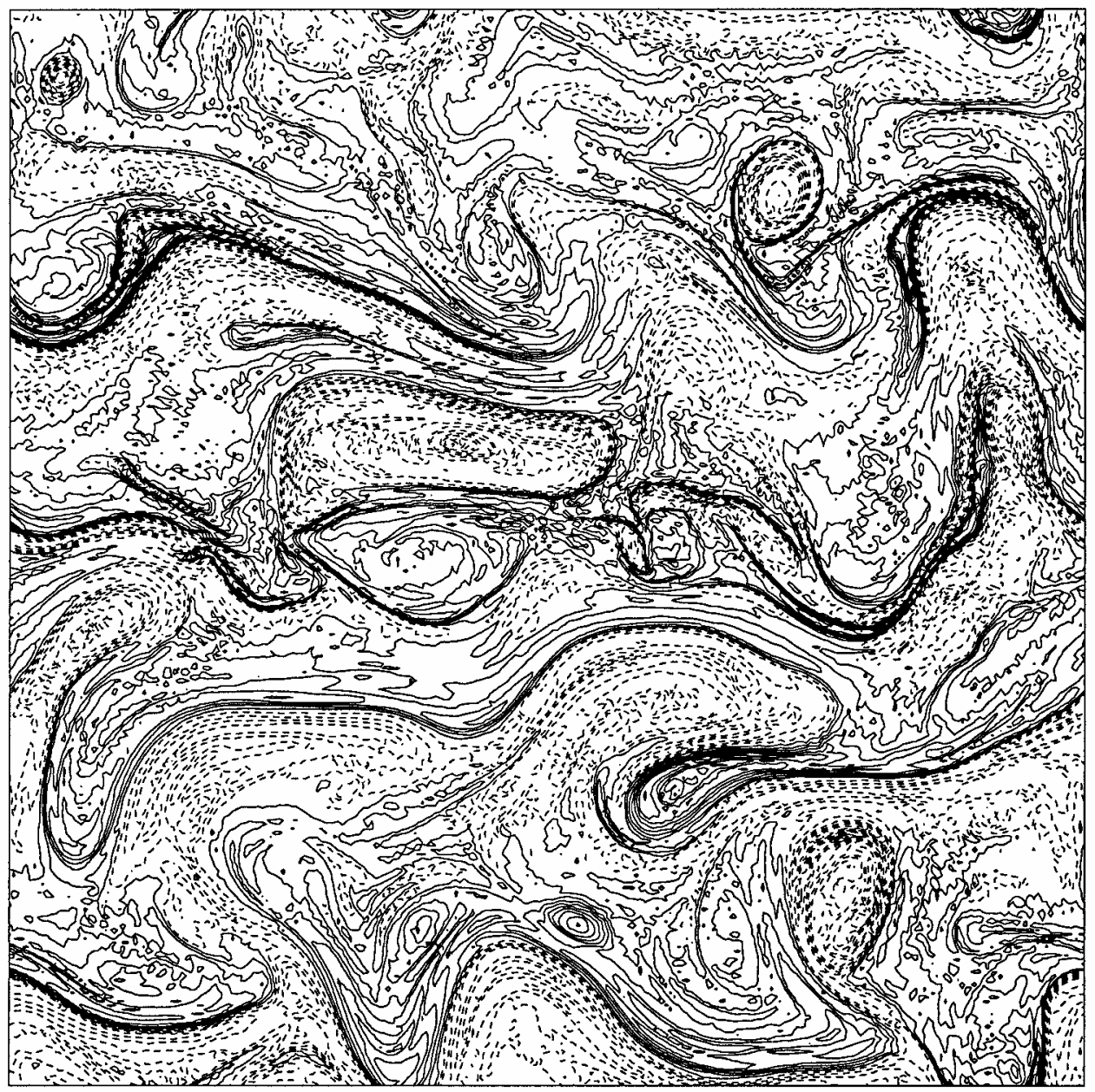

\section{CONTOUR FROM -90 TO 80 BY 10}

Figure 2. Contour map of the vorticity field 7 days after the initial time. Dashed and continuous contours correspond, respectively, to negative and positive values relative to the mean.

by much smaller structures which are mostly anisotropic, whose extrema are now located near strong vorticity fronts and in the cores of small-scale coherent vortices. The correlation with the vorticity field is again significant, but the width of the NIO structures is smaller than that of mode- 3 structures and their geometries are different. The NIO speed of mode 7 (Fig. 4b) reveals a sharp gradient of NIO kinetic energy in regions of intense vorticity fronts, with a strong depletion of NIO energy on the positive vorticity side and a concentration of NIO energy on the negative vorticity side, which is reminiscent of the 
(a) $\operatorname{IM}(\mathrm{NIO})$ of mde 3 at $t=7$. days

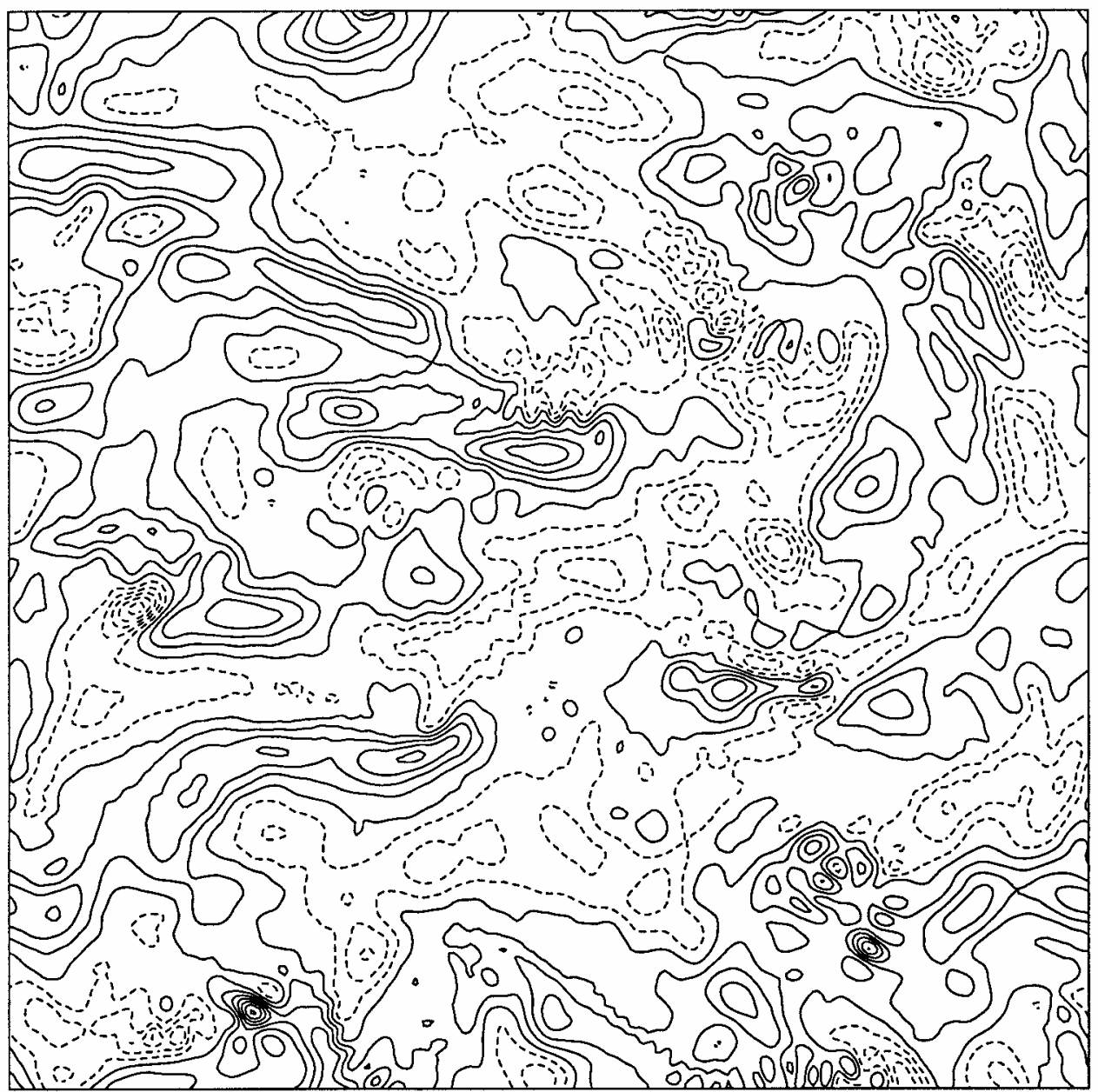

\section{CONTOUR FROM -2.5 TO 3 BY .5}

Figure 3. Contour maps of the imaginary part $(\operatorname{Im}(\mathscr{L} A))$ of NIO amplitude (a) and NIO speed $\left(\sqrt{u^{2}+v^{2}}=|\mathscr{L A}|\right)$; (b) associated with the 3rd mode, 7 days after the initial time. Dashed and continuous contours correspond, respectively, to negative and positive values relative to the mean.

'trapping' regime. The corresponding energetic structures are mostly thin filamentary structures. NIO kinetic energy is expelled from the small-scale cyclonic vortices, and concentrated in small-scale anticyclonic vortices. For both lower and higher modes, the departure from the initial homogeneous field is quite rapid: after 3 days, the spatial distribution is similar to that after 7 days but with half the amplitude. This emphasizes the short time scale associated with the effects of the QG flow on NIOs. 
(b) NIO speed of mde 3 at $t=7$. days

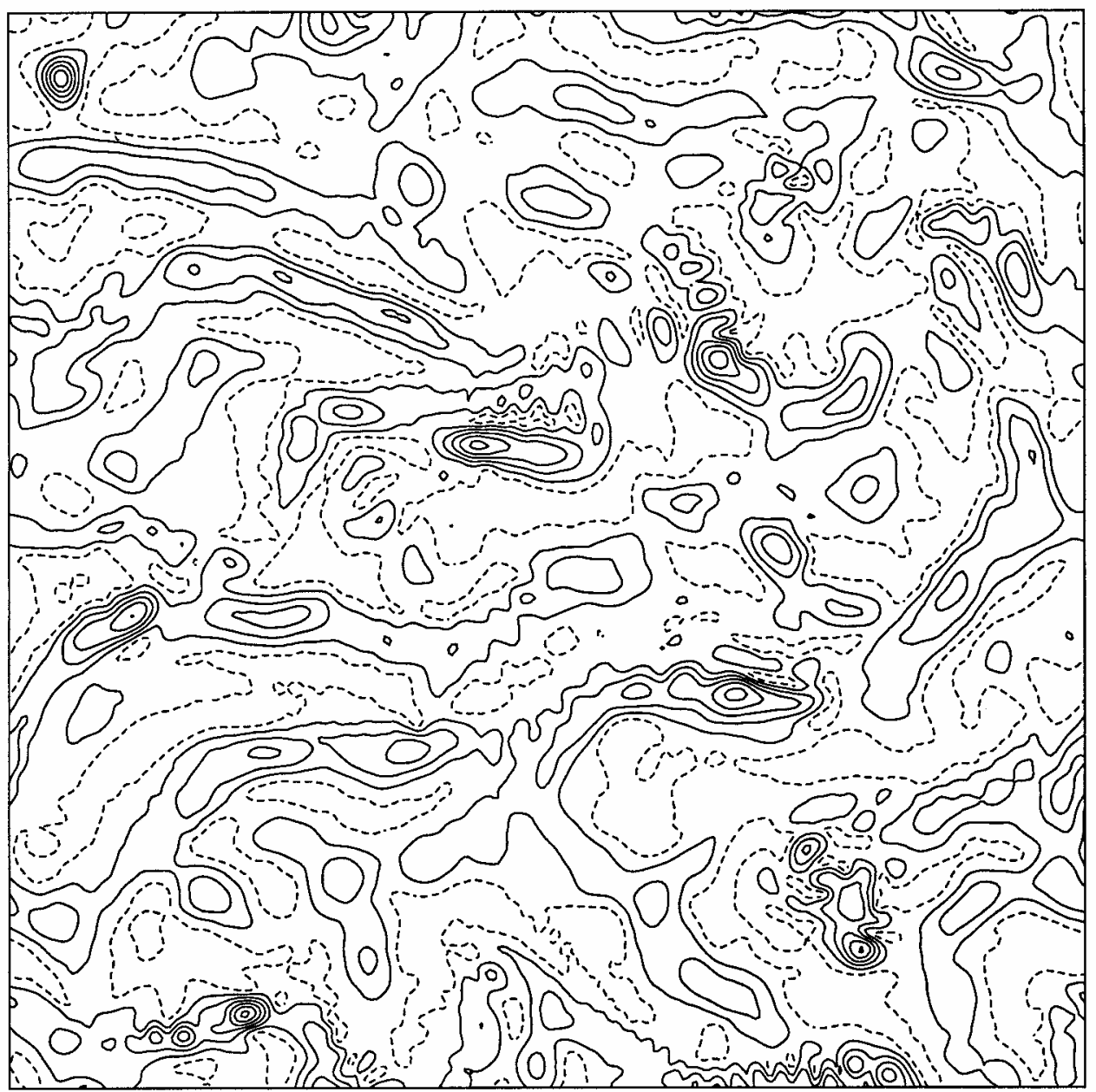

\section{CONTOUR FROM -.5 TO 2.5 BY .5}

Figure 3. (Continued)

Figure 5 and subsequent figures show the spectra of modes 3 (with Rossby radius $16 \mathrm{~km}$ ) to 15 (with Rossby radius $2.4 \mathrm{~km}$ ). These spectra reveal a sharp discontinuity of the spectral slope at a wavenumber, $k_{s}$, which we shall call specific wavenumber, which depends on the mode. The specific wavenumbers of lower modes are smaller than those of higher modes. For wavenumbers lower than the specific wavenumber, the spectra of the different baroclinic modes are almost identical and display a $k^{-1.5}$ spectral slope. For higher wavenumbers, amplitudes are smaller for lower modes than for higher modes and the spectra display a $k^{-5.5}$ slope. These two spectral slopes are close to the vorticity 
(a) IM(NIO) of mde 7 at $t=7$. days

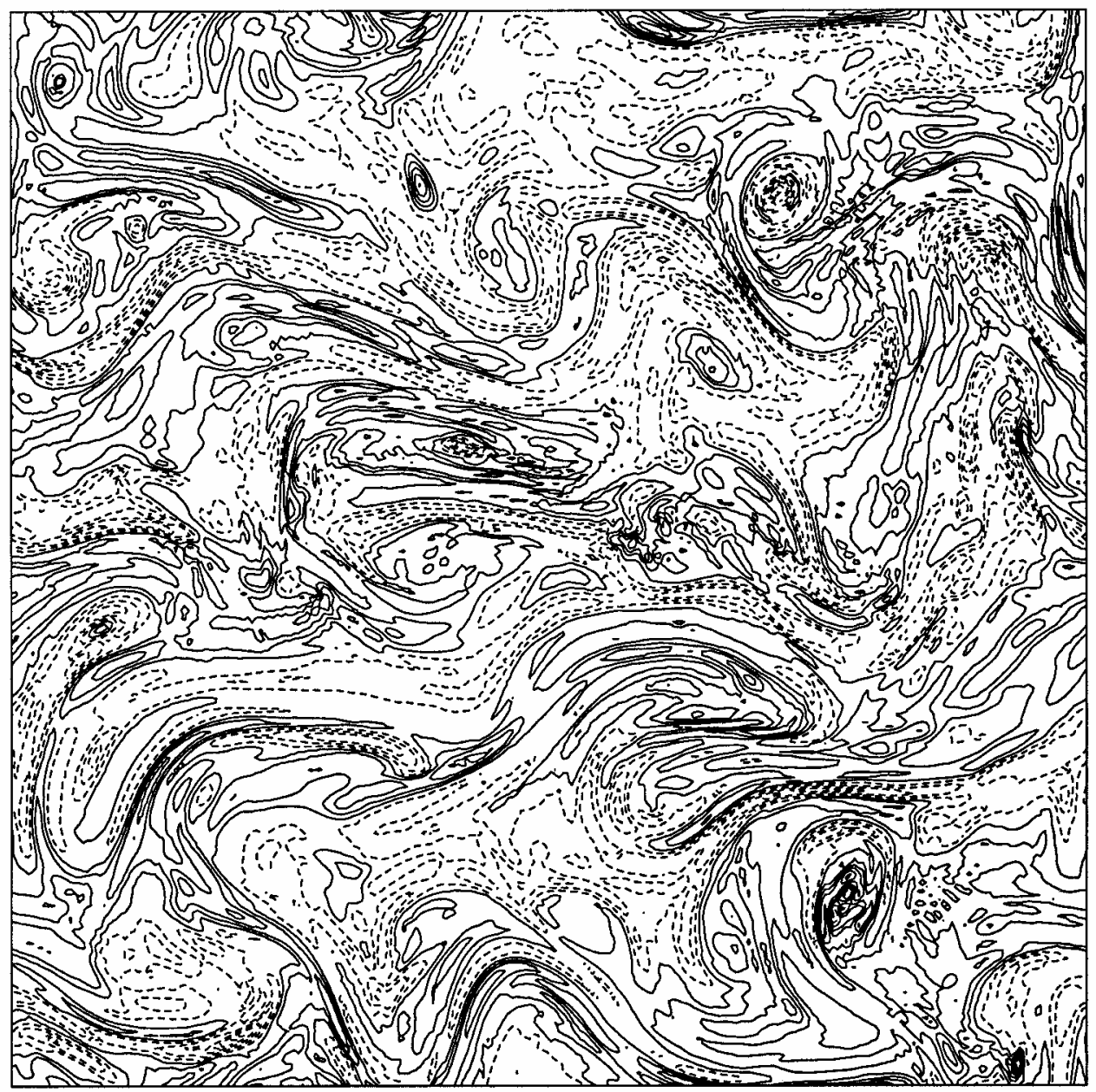

\section{CONTOUR FROM -3.5 TO 2.5 BY .5}

Figure 4. Contour maps of the imaginary part $(\operatorname{Im}(\mathscr{L} A))$ of NIO amplitude (a) and NIO speed $\left(\sqrt{u^{2}+v^{2}}=|\mathscr{L A}|\right)$; (b) associated with the 7 th mode, 7 days after the initial time. Dashed and continuous contours correspond, respectively, to negative and positive values relative to the mean.

$\left(k^{-1.5}\right)$ and streamfunction $\left(k^{-5.5}\right)$ slopes, which suggests that the large-scale (respectively small-scale) NIO field is correlated to the large-scale vorticity field (respectively the small-scale streamfunction field). This result is reminiscent of previous results (see Klein and Treguier, 1995a and YBJ) on the 'trapping' and 'strong dispersion' regimes that should characterize the dynamics of the large and small NIO scales, respectively. The time evolution of the spectra displays another interesting feature: for $6<k<k_{s}$ spectral 
(b) NIO speed of mde 7 at $t=7$. days

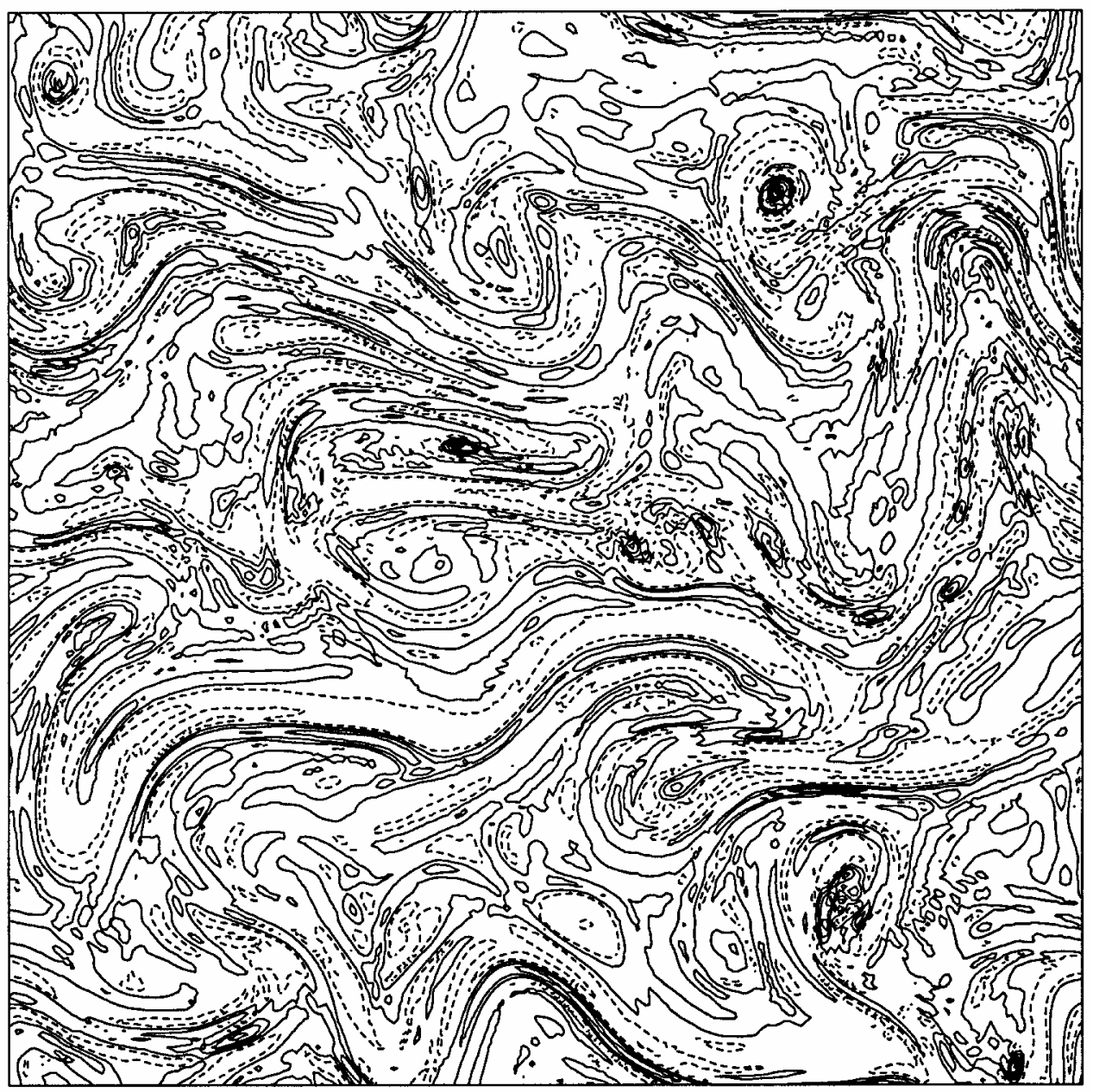

CONTOUR FROM -.5 TO 4 BY .5

Figure 4. (Continued)

amplitudes grow with time for all modes while for $k>k_{s}$ the spectral amplitude of each mode is steady. As a result, the specific wavenumbers decrease with time for all baroclinic modes. Later, the spectral amplitude still increases in time, although weakly and only in the $6<k<k_{s}$ region (compare Fig. 5a and Fig. 5b). Figure 6 shows the spectra of kinetic energy in the NIO baroclinic modes (using (7) integrated over $H$ ). These spectra display a maximum near $k_{s}$, which corresponds to the dominant wavelength of the NIO waves. This means that the kinetic energy of NIO modes is concentrated in scales close to $k_{s}^{-1}$.

Thus, the nature of the horizontal dispersion of NIOs induced by a turbulent mesoscale 

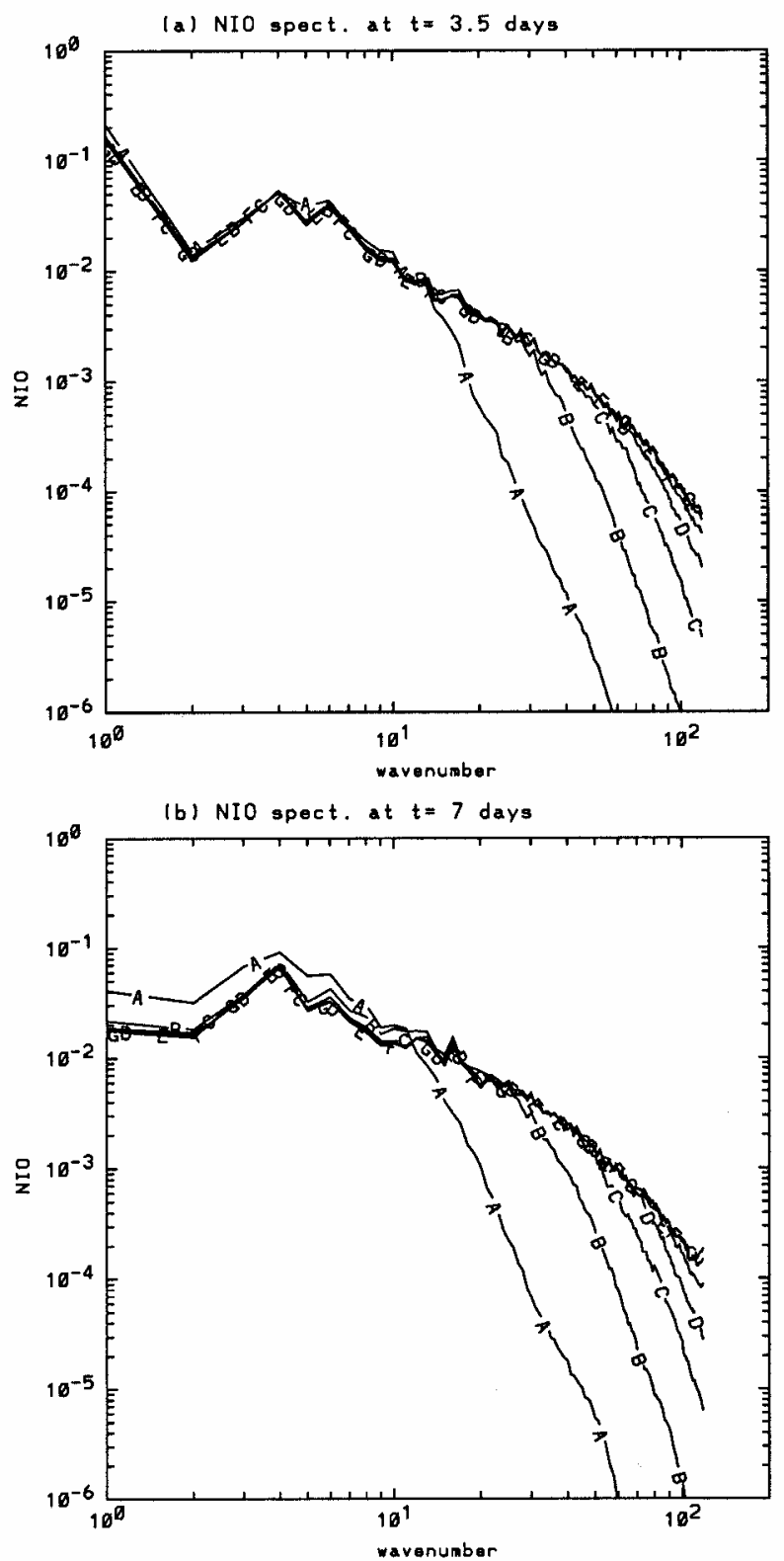

Figure 5. Spectra of the NIO baroclinic modes 3, 5, 7, 9, 11, 13, 15 (respectively, A to G) after a simulation duration of 3.5 days (a) and 7 days (b).

eddy field depends on whether large or small scales are examined. The 'trapping' regime affects larger, energetic, scales, and the 'strong dispersion' regime affects smaller, less energetic, scales. The horizontal wavenumber that separates these two regions is lower for 

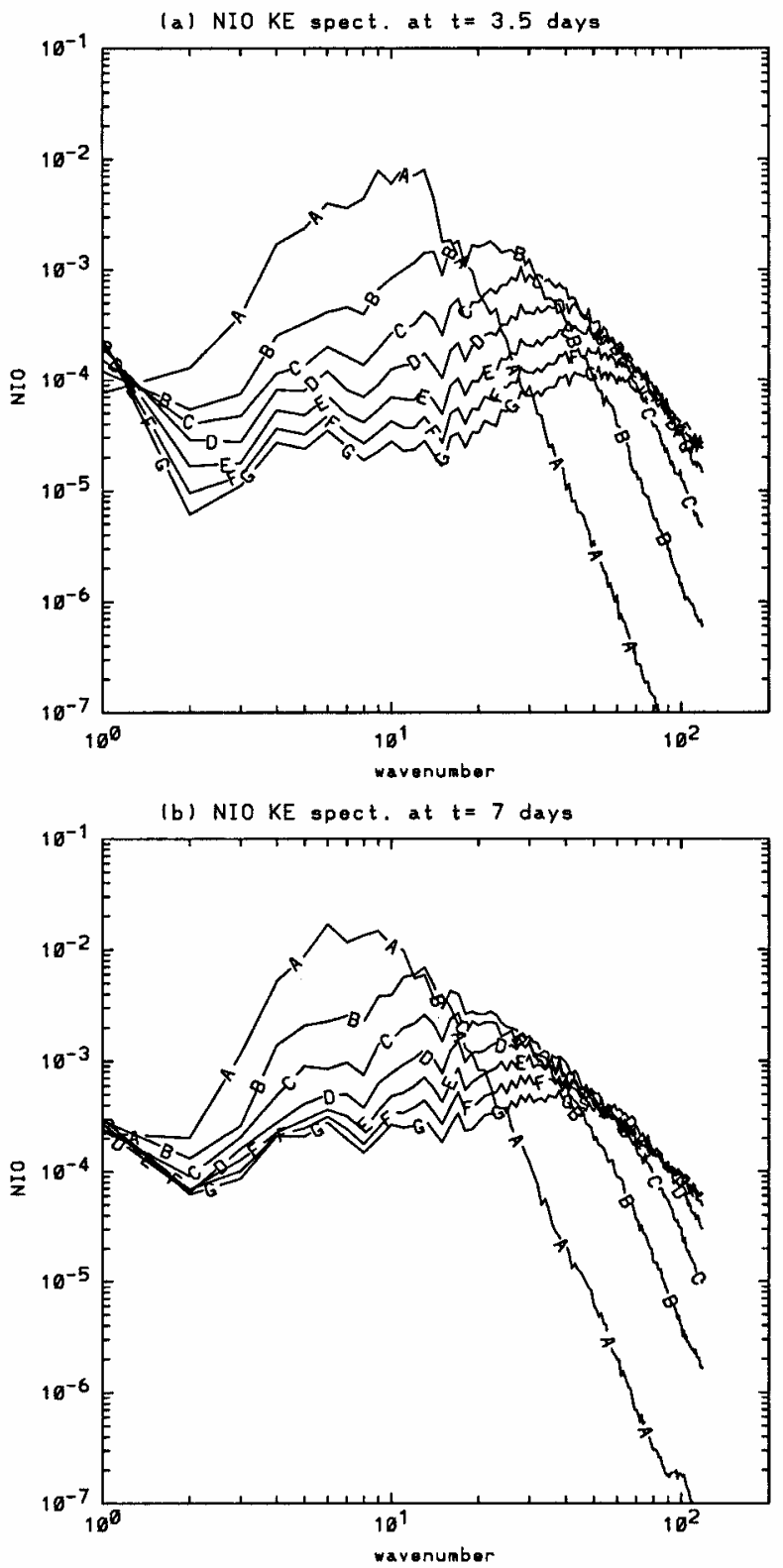

Figure 6. Kinetic energy spectra of the NIO baroclinic modes 3, 5, 7, 9, 11, 13, 15 (respectively, A to G) after a simulation duration of 3.5 days (a) and 7 days (b).

lower baroclinic modes and higher for higher modes. Furthermore, it varies with time, becoming smaller as time increases. One might wonder whether, for very long times, the 'strong dispersion' regime could affect all scales for the lower modes, in which case the 
spatial structure of those modes would resemble the streamfunction field. A long numerical simulation (over 21 days) reveals saturation that stops the decrease of this specific wavenumber near the peak of the vorticity spectrum (i.e., $k=5$ ).

These new features displayed by the numerical results raise several questions. How may we explain the spatial structure of the NIOs, in particular the sharp discontinuity in the NIO spectral slope? Is there a typical length scale for NIO kinetic energy structures resulting from dispersion by the mesoscale eddy field? Which dispersion regime dominates in those structures, the 'strong dispersion' regime or the 'trapping' regime?

\section{Analysis using a simplified NIO equation}

Additional numerical simulations have been performed without the advective terms in (14). Results in both physical and spectral spaces are similar to those obtained with the full NIO equation. Comparing Figure 5a and Figure $7 \mathrm{~b}$ reveals that, for each mode, the discontinuity in the spectral slope occurs at the same specific wavenumber. Thus, the NIO evolution is mostly driven by the refraction term (involving the vorticity) and the dispersion term present in (14) (respectively the third and fourth term of (14)). To understand the appearance of the discontinuity in the spectral slope, we examine the NIO equation in Fourier space after further simplifying the refraction term. Using

$$
X(x, y, t)=\sum_{p=-N}^{p=N} \sum_{q=-N}^{q=N} \hat{X}_{p q}(t) e^{i(p x+q y)},
$$

with $X$ any variable, $p$ and $q$ the horizontal wavenumbers and $N$ the number of horizontal modes considered, (14) discarding advection and diffusion becomes (subscript $m$ is dropped hereafter):

$$
\frac{d \widehat{\mathscr{L A}}_{p q}}{d t}+\frac{i}{2} \frac{k^{2} \widehat{\mathscr{L A}}_{p q}}{\lambda^{2}}+\frac{i}{2} \sum_{p=p_{1} \pm p_{2}} \sum_{q=q_{1} \pm q_{2}} \hat{Z}_{p 1 q_{1}} \widehat{\mathscr{L A}}_{p 2 q_{2}}=0 .
$$

with $k^{2}=p^{2}+q^{2}$. Initially, from (16), $\mathscr{L A}$ is a large-scale field such that $\left|\widehat{\mathscr{L A}}_{10}\right|=1$ and $\left|\widehat{\mathscr{L A}}_{p q}\right|=0$ otherwise. We assume that there is a spectral gap between the initial large-scale field and the $\mathscr{L A}$-scales produced later and that $\left|\widehat{\mathscr{L A}}_{p q}\right| \ll\left|\widehat{\mathscr{L A}}_{10}\right|$ for $p, q>2$. Such a gap is observed during early times (the first two days) of the numerical simulations. From this assumption, the refraction term can be approximated as:

$$
\sum_{p=p_{1} \pm p_{2}} \sum_{q=q_{1} \pm q_{2}} \hat{Z}_{p_{1} q_{1}} \widehat{\mathscr{L A}}_{p_{2} q_{2}} \approx \sum_{p=p_{1} \pm 1} \hat{Z}_{p_{1} q} \widehat{\mathscr{L A}}_{10} \approx \hat{Z}_{p q} \widehat{\mathscr{L A}}_{10}
$$

where the last near-equality is due to the fact that we are interested in the higher components of $\mathscr{L} A$, where the difference between $\hat{Z}_{p \pm 1, q \pm 1}$ and $\hat{Z}_{p q}$ is unimportant. The resulting NIO spectral equation decouples for each wavenumber: 


$$
\frac{d \widehat{\mathscr{L A}}_{k}}{d t}+\frac{i}{2} \frac{k^{2} \widehat{\mathscr{L A}}_{k}}{\lambda^{2}}+\frac{i}{2} \hat{Z}_{k} \widehat{\mathscr{L A}}_{10}=0
$$

where $A_{k}$ is shorthand for $A_{p q}$.

We now examine the analytical solution of (20) to explain the appearance of the discontinuous spectral slope and to characterize the different dispersion regimes involved. Using zero initial condition, the solution is:

$$
\left|\widehat{\mathscr{L A}}_{k}\right|^{2}=2|\hat{Z}|^{2}\left|\widehat{\mathscr{L A}}_{10}\right|^{2} \frac{\lambda^{4}}{k^{4}}\left[1-\cos \left(\frac{k^{2}}{2 \lambda^{2}} t\right)\right],
$$

with period

$$
T(k)=\frac{4 \pi \lambda^{2}}{k^{2}},
$$

that depends on the horizontal wavenumber $k$ and is directly related to the dispersion effects.

We focus on the spectral region corresponding to $k>5$ since the slope discontinuities occur mostly in that region. In that part of the spectrum, the vorticity can be approximated as:

$$
|\hat{Z}|^{2}(k) \approx \alpha_{z}^{2} k^{-1.5}
$$

with $\alpha_{z}$ a constant. In order to examine the properties of $\left|\widehat{\mathscr{L A}}_{k}\right|^{2}$ we define a critical wavenumber, $k_{c}$, that follows from (21):

$$
k_{c}^{2}=\frac{\pi \lambda^{2}}{t} .
$$

Now, at a given time $t$, the simplified solutions of $\left|\widehat{\mathscr{L A}}_{k}\right|^{2}$ for $k=k_{c}, k<k_{c}$ and $k>k_{c}$ are the following:

- For $k=k_{c}$, the solution equals the average value:

$$
\left|\widehat{\mathscr{L A}}_{k_{c}}\right|^{2}=2|\hat{Z}|^{2}\left|\widehat{\mathscr{L A}}_{10}\right|^{2} \lambda^{4} k_{c}^{-4} \approx 2\left|\widehat{\mathscr{L A}}_{10}\right|^{2} \alpha_{z}^{2} \lambda^{4} k_{c}^{-5.5} .
$$

For this particular wavenumber, this solution expresses that an exact equilibrium between dispersion and refraction processes (expressed respectively by the second and third terms in (20)) has been attained.

- For $k<k_{c}$ (starting from $k=k_{c} \sqrt{2} / 2$ ), the period of the solution is such that $T(k) \gg t$ (using (22) and (24)). Consequently, at time $t$, the amplitude can be approximated by:

$$
\left|\widehat{\mathscr{L A}}_{k}\right|^{2} \approx \frac{|\hat{Z}|^{2}\left|\widehat{\mathscr{L A}}_{10}\right|^{2}}{4} t^{2} \approx \frac{\left|\widehat{\mathscr{L A}}_{10}\right|^{2} \alpha_{z}^{2}}{4} k^{-1.5} t^{2}
$$


In this part of the spectrum, the solution is growing with time and is proportional to the vorticity, $\hat{Z}(k)$, and the background NIO, $\widehat{\mathscr{L A}}_{10}$. Dispersion processes are weak and act to concentrate NIO energy in negative vorticity regions. This corresponds to the 'trapping' regime. In this part of the spectrum, the spatial structure of the NIO field is imposed by the vorticity field and the solution behaves like $k^{-1.5}$.

- For $k>k_{c}$ (starting from $k=k_{c} \sqrt{2}$ ), the period of the solution is such that $t \gg$ $T(k)$. The NIO solution experiences oscillations (with a period that decreases as $k$ increases) about a mean value of:

$$
\left|\widehat{\mathscr{L A}}_{k}\right|^{2}=2|\hat{Z}|^{2}\left|\widehat{\mathscr{L A}}_{10}\right|^{2} \frac{\lambda^{4}}{k^{4}} \approx 2\left|\widehat{\mathscr{L A}}_{10}\right|^{2} \alpha_{z}^{2} \lambda^{4} k^{-5.5}
$$

This mean value behaves like $k^{-5.5}$ and corresponds to the 'strong dispersion' regime of YBJ. The spectral slope is that of the streamfunction spectrum. The oscillation around this mean value corresponds to NIO energy concentration (when the refraction term in (20) overcomes the dispersion term) followed by an NIO energy relaxation (when the dispersion term in (20) overcomes the refraction term).

We now examine at which scale dispersive processes are most efficient. Dispersion term in (20) can be characterized by $D_{t}=k^{2} \widehat{\mathscr{L A}}_{k} /\left(2 \lambda^{2}\right)$, and (25) and (26) lead to:

$$
\left|D_{t}\right|^{2} \approx\left\{\begin{array}{ll}
\frac{\left|\widehat{\mathscr{L A}}_{10}\right|^{2} \alpha_{z}^{2}}{16 \lambda^{4}} t^{2} k^{2.5} & \text { if } k<k_{c} \\
\frac{\left|\widehat{\mathscr{L A}}_{10}\right|^{2} \alpha_{z}^{2}}{2} k^{-1.5} & \text { if } k>k_{c}
\end{array} .\right.
$$

These relations show that the spectrum of dispersion is characterized by a spectral peak at $k \approx k_{c}$. So the maximum efficiency of dispersion occurs at $k=k_{c}$. In consequence, the critical wavenumber $k_{c}$ should characterize the scale of the structures where NIO energy is concentrated as shown in the numerical simulations (see Fig. 6). This important property is due to the fact that the spectral slope of vorticity is gentler than $k^{-4}$.

This analysis explains the shape of the numerical NIO spectrum, in particular the existence of a discontinuity of the spectral slope. It also yields a simple estimate of the critical wavenumber that characterizes this discontinuity. As shown in the next section, this critical wavenumber, $k_{c}$, is quite close to the specific wavenumber, $k_{s}$, of the numerical results. From (24), for a given wavenumber, dispersion is equal to refraction at the end of the first phase. This should affect first the highest wavenumbers. If $k_{N}$ designates the highest wavenumber, dispersion equals refraction at time $t=\pi \lambda^{2} k_{N}^{-2}$. 


\section{Discussion}

The preceding analysis does not take into account processes such as the wave-wave interactions between the NIO subinertial amplitude, $\mathscr{L A}$, and background vorticity, $Z$, that lead to nonlinear energy transfer between the NIOs horizontal modes. These interactions have been assessed using two simulations, one corresponding to (20) and one to (14) with the advection terms suppressed. Comparing the two for a given baroclinic mode reveals little change of the spatial heterogeneity properties of the NIO fields during the first 2 days. After 3.5 days, both fields are still close, and the only change is the larger amplitude of the NIO field obtained with (20). Analysis of the NIO spectra (see Fig. 7) reveals that these interactions are non-negligible after 3.5 days. They reduce the amplitude at large scales $\left(k<k_{s}\right)$, while small scales $\left(k>k_{s}\right)$ are unchanged. A more careful examination shows that they reduce the amplitude of the $Z \mathscr{L} A$ term in the NIO equation, which is still acting to force $\mathscr{L} A$ through the vorticity $Z$. The high correlation of $\mathscr{L} A$ with $Z$ is unchanged. Thus, these wave-wave interactions do not change the nature of the dynamical regimes identified in the preceding analysis. The specific wavenumber $k_{s}$ is slightly larger than $k_{c}$ (given by (24)), and $k_{c}$ is a lower limit for $k_{s}$. For large times, the wave-wave interactions are responsible for the saturation that stops the evolution of $k_{s}$.

Comparison of the reference simulation described in Section 3 (cf. Figs. 5 and 6) with the simulation with the advection term suppressed (cf. Fig. 7b) reveals that advection processes have an essentially negligible effect during the first 3 days. Later, these processes become non-negligible, although they do not greatly affect the spatial heterogeneity of the NIO during the next 4 days: the direct cascade induced by advection slows the rate of decrease of the specific wavenumber $k_{s}$. These cascade effects also amplify the smallest NIO scales. As a result, the NIO spectral slopes are slightly gentler at high wavenumber. In physical space, this makes the small-scale NIO structures more stretched.

The good correspondence between the simple analytical findings and the different numerical simulations suggests that $k_{c}$, given by (24), is a reasonable estimate of the specific wavenumber $k_{s}$, and thus of the characteristic scale of the structures at which NIO kinetic energy becomes concentrated. This estimate should be considered as a lower limit. The preceding analytical analysis also highlights the physical mechanisms that drive the evolution of the NIOs embedded in a QG flow field. In summary, the vorticity structures of different sizes produce NIO spatial structures of the same size with an amplitude proportional to that of the vorticity. However, the effects of the dispersion mechanisms concentrate the NIO kinetic energy in structures with scales close to $k_{c}^{-1}$. For these specific spatial structures, the vorticity effects equilibrate the dispersion effects. A simple physical interpretation is suggested by Metzger (1999), who characterized the NIO equation for a simple case by a "dispersivity" parameter analogous to the diffusivity associated with passive scalar diffusion processes. From the nondimensional NIO equation (14), the dispersivity associated with the $m$ th 

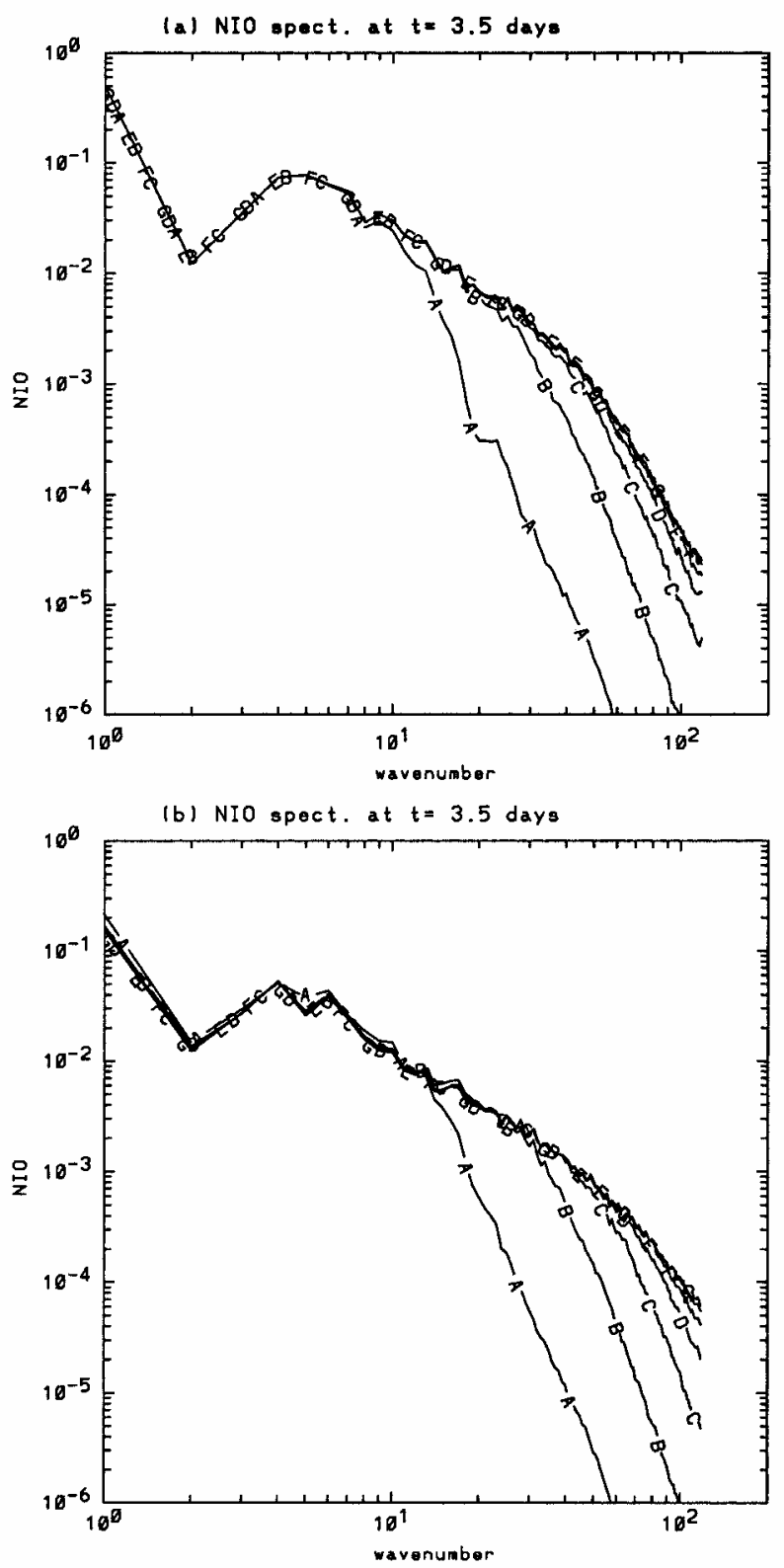

Figure 7. Spectra of NIO amplitude of the baroclinic modes 3, 5, 7, 9, 11, 13, 15 (respectively, A to G), 3.5 days after the initial time, obtained with a simulation corresponding to (20) (a) and with a simulation corresponding to (14) with the advection terms suppressed(b).

baroclinic mode is $h_{m}=\lambda_{m}^{-2}$. Then the length scale ( $\delta$ ) over which the tracer is dispersed is given by $\delta^{2}=\pi h_{m} t$. This is the scale given by $k_{c}$ (see (24)). The dimensional forms of $k_{c}$ and $\delta^{2}$ are $k_{c}^{2}=\pi / f_{0} t R_{m}^{2}$ and $\delta^{2}=\pi f_{0} t R_{m}^{2}$, respectively. 


\section{Conclusion}

The present study sheds new light on NIO dispersion in a mesoscale eddy field. The trapping regime dominates the dispersion of the NIOs, with the important consequence that the NIO spatial distribution matches the QG vorticity field more closely than the streamfunction field. This is apparent in Fig. $8(\mathrm{a}-\mathrm{b})$ which shows the vorticity and streamfunction fields filtered to retain only Fourier modes $k<k_{s}$ (with $k_{s}$ corresponding to the specific wavenumber of the 3rd baroclinic mode). Comparison with Figure 3(a-b) shows that the NIOs more closely resemble the vorticity field.

This study also reveals the existence of a specific wavenumber, $k_{s}$, that separates the large-scale NIO structures where the 'trapping' regime dominates from the less energetic small-scale NIO structures where the 'strong dispersion' regime dominates. The usual interpretation (Kunze, 1985) would be that lower horizontal wavenumber $k$ implies frequencies closer to $f$ which would be more susceptible to vorticity trapping while high $k$ implies higher frequencies which will be freer to disperse. However, the present results reveal a conspicuous discontinuity in the dynamical regimes, which occurs at $k=k_{s}$. The small-scale $\left(k>k_{s}\right)$ NIO structures do not grow in time, while the large-scale $\left(k<k_{s}\right)$ structures are active and grow, at least in the initial linear regime (Fig. 5a-b). The efficiency of the dispersion mechanisms for scales close to $k_{s}$ leads to a strong concentration of NIO kinetic energy at these scales. This feature is due to the competition between the effects of vorticity structures and the dispersion of NIO structures that have the same horizontal scale $\left(k_{s}^{-1}\right)$. NIO energy associated with the higher vertical modes is mostly found in small-scale negative vorticity structures like filaments near the sharpest vorticity fronts, while the NIO energy associated with the lower modes is concentrated within the mesoscale anticyclonic eddies. For large times, saturation stops the time evolution of $k_{s}$ at a value close to the kinetic energy spectrum peak of the QG flow field.

One important consequence is that the length scale characterizing the most energetic NIO structures increases with time, and this increase is faster for the lower modes. Thus, the QG structures that significantly affect the NIO energy of a given baroclinic mode differ (with respect to both scale and location in physical space) from those that affect the NIO energy of other modes. These results are important since a turbulent mesoscale eddy field cannot be considered as a simple juxtaposition of geometrical structures with different length scales: in such a field, small-scale structures are embedded in larger structures. Since the vertical propagation of NIO energy results from the combination of different baroclinic modes, this should lead to a 3-D distribution of the near-inertial oscillations quite different from that resulting from each QG structure independent of the others.

As mentioned in the introduction, this study is a first step toward understanding 3-D NIO dispersion in a fully turbulent mesoscale eddy field. Here we have focused mostly on the horizontal dispersion of the different NIO baroclinic modes in a situation that corresponds to a summertime stratification for which the vertical propagation of the NIOs is very weak (see also Klein and Treguier, 1995a). Such a situation makes the nonlinear energy transfer between the different NIO baroclinic modes to be negligible and thus prevents the 
(a) PHI at $z=100 \mathrm{~m}$ and $t=7$. days

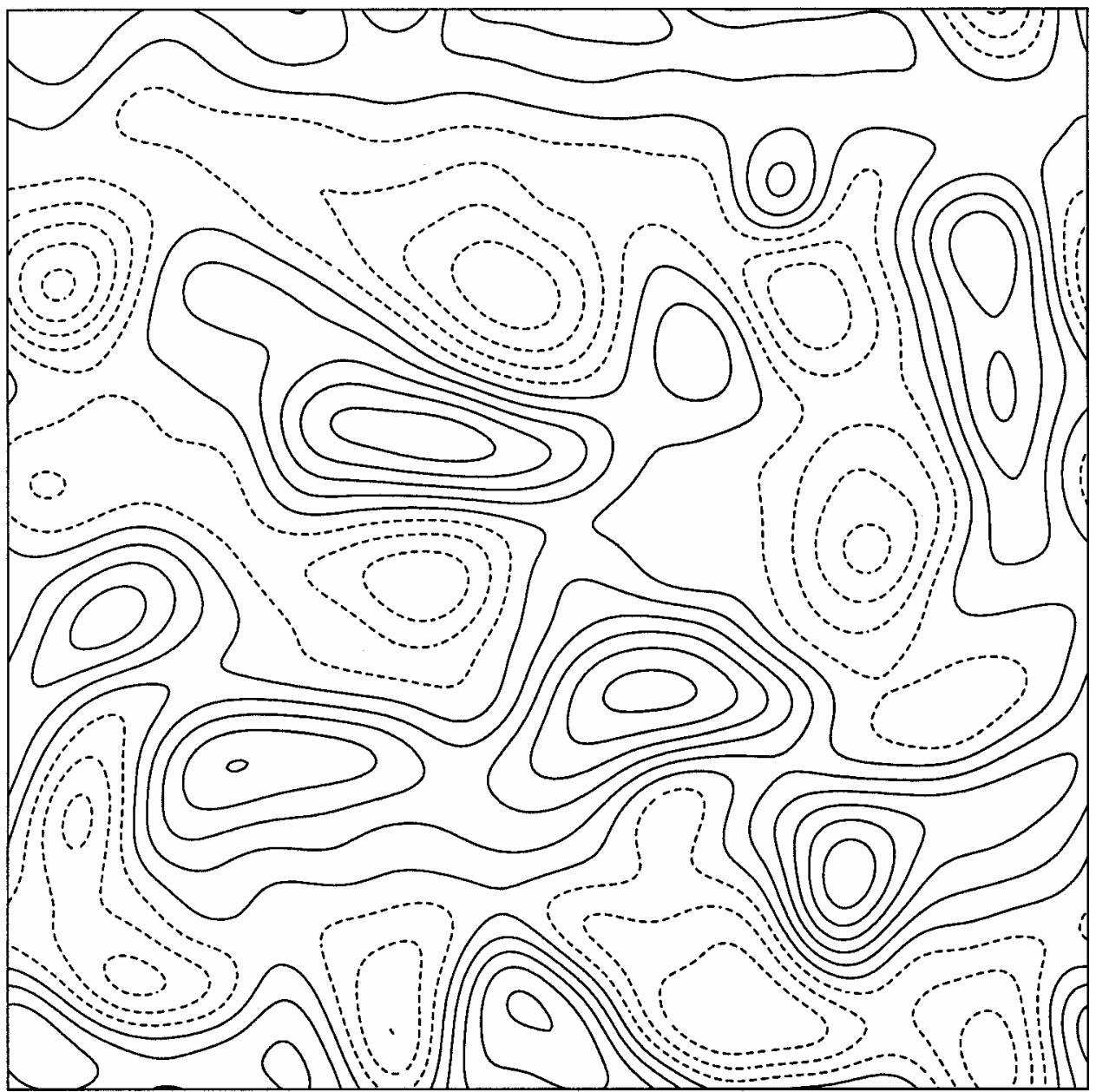

\section{CONTOUR FROM -2.5 TO 2.5 BY .5}

Figure 8. Contour map of the filtered streamfunction (a) and vorticity (b) with $k<k_{s}$ (with $k_{s}$ corresponding to mode 3 ) after 7 days.

appearance of vertical critical layers at least for the timescales considered. It is equivalent to assuming that the QG flow is barotropic. However for a wintertime stratification, corresponding to a large mixed-layer depth, the approach using a vertical normal mode expansion is no longer valid when a baroclinic QG flow is present, even for a short time-duration of 15 days. In this situation nonlinear energy transfer between baroclinic NIO modes may affect their horizontal dispersion and lead to the appearance of vertical critical layers (see for example Eriksen (1993) for a description of the internal-wave modal 
(b) $\mathrm{Z}$ at $\mathrm{z}=100 \mathrm{~m}$ and $\mathrm{t}=7$. days

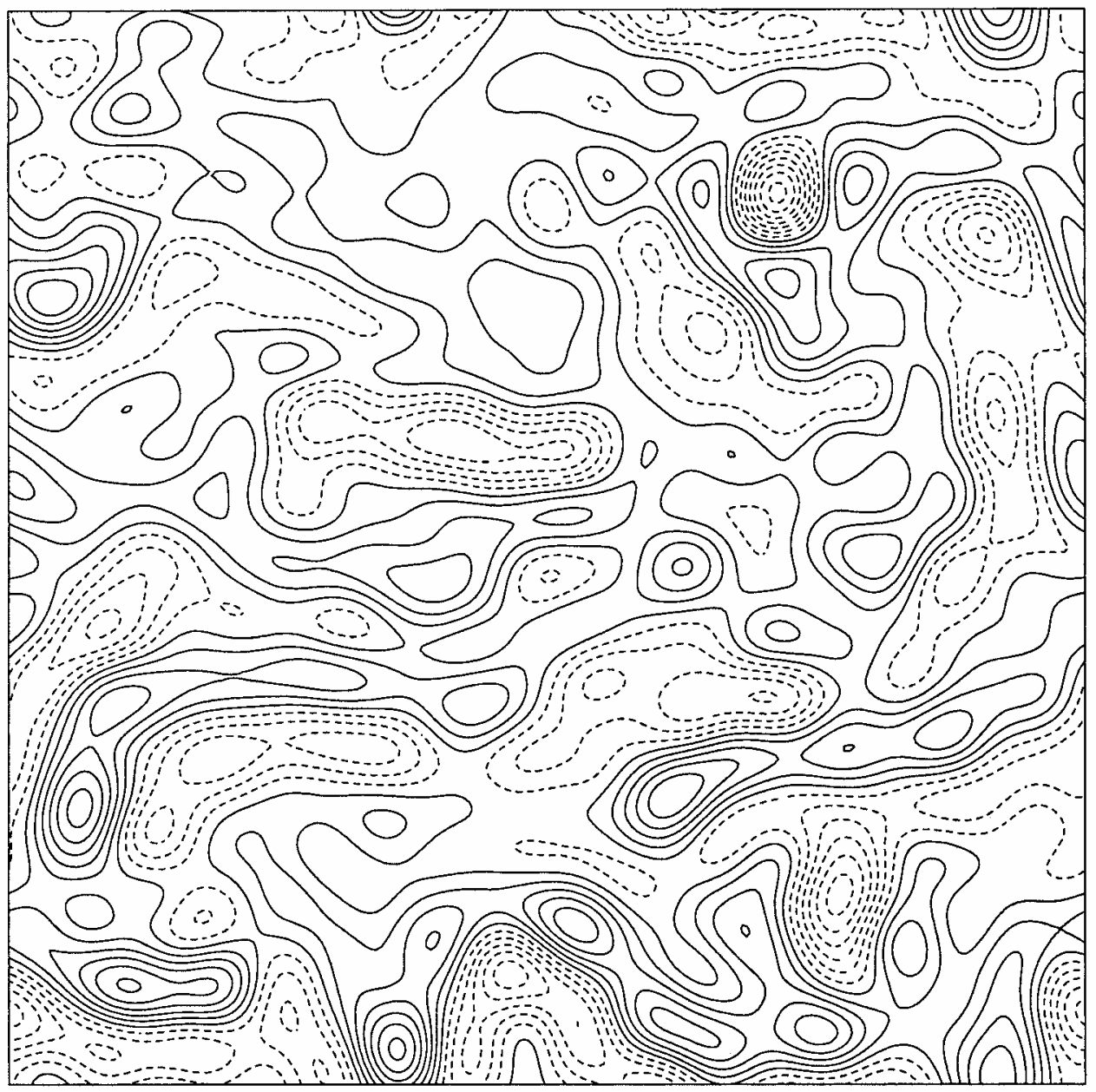

Figure 8. (Continued)

response to wind-forcing as function of mixed-layer thickness). Two extensions of this work, for a wintertime stratification, should be undertaken. The first concerns the implications of the present results on the vertical propagation of NIOs embedded in a barotropic QG flow. This requires an approach using a much higher resolution in the vertical and based on the examination of individual structures. Although cpu intensive, this extension can be handled within the YBJ framework. The second is to consider the effects of the nonlinear energy transfers between different NIO baroclinic modes that occur through the vorticity and advection terms when the QG flow is baroclinic. In this situation a 
preliminary study of the time scale involved in the appearance of vertical critical layers should be undertaken.

Another direction to explore is the role of the wave-wave interactions. The NIO equation (8) is based on the assumption that they are negligible, which is justified from the scalings used. However some previous studies have produced arguments showing that, even when the NIO Rossby number is formally infinite, these interactions are still negligible. Klein and Treguier (1995a) found that simulations over 20 inertial periods with and without these nonlinear terms produced identical results. In another study, Klein and Treguier (1995b) investigated the role of these interaction terms more closely. Both numerical and asymptotic analysis showed that these terms cancel. Nevertheless one may wonder whether, when NIOs concentrate in small-scale vorticity structures, interaction terms could become significant and be a sink for trapped inertial energy. Again, previous results indicate that this is not the case. McComas and Muller (1981) and Kunze (1985) argue that the energy exchange with the mean flow or with the higher frequency internal waves does not provide a sink for trapped near-inertial wave energy. Kunze et al. (1995) described observations in a warm ring vortex that convincingly demonstrated this point. Shear instability still remains the most plausible sink. However, a quantitative test should be undertaken to better understand the role of these terms.

Acknowledgments. This work was supported by IFREMER, the CNRS and the Programme on Transport Processes in the Atmosphere and Oceans of the European Science Foundation. Some of the calculations reported here were done on the CRAY C90 and NEC SX5 of the IDRIS (Orsay, France). Conversations with Bill Young were very helpful. We thank both reviewers for their comments and suggestions.

\section{REFERENCES}

Balmforth, N. J., S. G. Llewellyn Smith and W. R. Young. 1998. Enhanced dispersion of near-inertial waves in an idealized geostrophic flow. J. Mar. Res., 56, 1-40.

D’Asaro, E. A. 1995a. Upper ocean inertial currents forced by a strong storm. Part II: Modeling. J. Phys. Oceanogr., 25, 2937-2952.

-1995b. Upper ocean inertial currents forced by a strong storm. Part III: Interaction of ocean currents and mesoscale eddies. J. Phys. Oceanogr., 25, 2953-2958.

D’Asaro, E. A., C. C. Eriksen, M. D. Levine, P. Niiler, C. A. Paulson and P. Van Meurs. 1995. Upper-ocean inertial currents forced by a strong storm. Part I: Data and comparisons with linear theory. J. Phys. Oceanogr., 25, 2909-2936.

Eriksen, C. C. 1993. Equatorial ocean response to rapidly translating wind bursts. J. Phys. Oceanogr., 23, 1208-1230.

Flierl, G. R. 1978. Models of vertical structure and the calibration of two-layer models. Dyn. Atmos. Oceans, 2, 341-381.

Gill, A. E. 1982. Atmosphere-Ocean Dynamics, Academic Press, 662 pp.

Hua, B. L. and D. B. Haidvogel. 1986. Numerical simulations of the vertical structure of quasi-geostrophicturbulence. J. Atmos. Sci., 43, 2923-2936.

Klein, P. and A. M. Treguier. 1995a. Dispersion of wind-induced inertial waves by a barotropic jet. J. Mar. Res., 53, 1-22.

1995b. Comments on "Scattering of inertial waves by an ocean front." J. Phys. Oceanogr., 25, 1018-1022. 
Klein, P., A. M. Treguier and B. L. Hua. 1998. Three-dimensional stirring of thermohaline fronts. J. Mar. Res., 56, 589-612.

Kunze, E. 1985. Near-inertial wave propagation in geostrophic shear. J. Phys. Oceanogr., 15, $544-565$.

Kunze, E. and T. B. Sanford. 1984. Observations of near-inertial waves in a front. J. Phys. Oceanogr., 14, 566-581.

Kunze, E., R. W. Schmitt and J. M. Toole. 1995. The energy balance in a warm-core ring's near-inertial critical layer. J. Phys. Oceanogr., 25, 747-761.

McComas, C. H. and P. Muller. 1981. The dynamics balance of internal waves. J. Phys. Oceanogr., 11, 970-986.

Metzger, M. M. 1999. Scalar dispersion in a two-dimensional random flow field, in Stirring and Mixing, 1999 Summer Study Program in Geophysical Fluid Dynamics, Woods Hole Oceanographic Institution, Woods Hole, MA.

Rubenstein, D. M. and G. O. Roberts. 1986. Scattering of inertial waves by an ocean front. J. Phys. Oceanogr., 16, 121-131.

Stammer, D. 1997. Global characteristics of ocean variability estimated from regional TOPEX/ POSEIDON altimeter measurements. J. Phys. Oceanogr., 27, 1743-1769.

Wang, D. P. 1991. Generation and propagation of inertial waves in the subtropical front. J. Mar. Res., 49, 619-633.

Webster, F. 1968. Observation of inertial-period motions in the deep sea. Rev. Geophys., 6, 473-490.

Weller, R. A. 1982. The relation of near-inertial motions observed in the mixed layer during the JASIN (1978) experiment to the local wind stress and to the quasi-geostrophic flow field. J. Phys. Oceanogr., 12, 1122-1136.

Wunsch, C. 1997. The vertical partition of oceanic horizontal kinetic energy. J. Phys. Oceanogr., 27, 1770-1794.

Young, W. R. and M. Ben Jelloul. 1997. Propagation of near-inertial oscillations through a geostrophic flow. J. Mar. Res., 55, 735-766.

Received: 19 June, 2000; revised: 20 July, 2001. 